\title{
Investigating Interfacial Parameters with Platinum Single Crystal Electrodes.
}

\author{
R. Martínez-Hincapié, P. Sebastián-Pascual, V. Climent, J.M. Feliu \\ Institute of Electrochemistry, University of Alicante,
}

Apartado 99. E-03080 Alicante, Spain

\begin{abstract}
The concepts of total and free charge of platinum single crystal electrodes are revised in this paper, together with the associated concepts of potential of zero total and free charge. Total charges can be measured from $\mathrm{CO}$ displacement method. Results on solution of different $\mathrm{pH}$ are described. A novel buffer composition is used to attain $\mathrm{pH}$ values close to neutrality while avoiding interferences from anion adsorption processes. Stress is made on the fact that free charges are not accessible through electrochemical measurement for systems at equilibrium since adsorption processes (hydrogen and hydroxyl) interfere with free charge determination. Still, a model is described that allows, under some assumptions, extract free charge values and the corresponding potential of zero free charge for Pt(111) electrodes. On the other hand, fast measurement outside equilibrium can separate free charges from adsorption processes based on their different time constant. In this way, the laser induced temperature jump experiment allows determination of the potential of maximum entropy, a magnitude that is intimately related with the potential of zero free charge. Values of the potential of maximum entropy as a function of $\mathrm{pH}$ are given for the different basal planes of platinum.
\end{abstract}

Keywords: Platinum single crystal, Electrochemical double layer, potential of zero total charge, potential of zero free charge, laser induced temperature jump, potential of maximum entropy

\section{Introduction:}

The potential of zero charge is a fundamental property of the electrified electrochemical interphase [1-3]. Its knowledge allows establishing a rational scale of potentials and, together with the knowledge of the differential capacity, it provides the relationship between charge and potential, the two key variables in electrochemical science. Such relationships are of paramount importance in the understanding of coulometric calculations.

Determination of the potential of zero charge for solid electrodes is usually attained by locating the minimum in the differential capacity in dilute solutions under the assumption of the validity of the Gouy-Chapman-Stern model of the double layer $[3,4]$. This procedure has been applied to the determination of the pzc of gold and silver electrodes[5-10]. However, this model is only valid in the absence of specific adsorption and therefore cannot be easily applied to more electroactive materials like platinum group metals ( $\mathrm{Pt}, \mathrm{Rh}, \mathrm{Ir}$ ). The thermodynamic description of the electrochemical interphase in contact with such metals has some particularities derived from the ability of these metals to adsorb hydrogen and anions. The classical thermodynamic analysis based on the electrocapillary equation has been more 
extensively applied to the ideally polarizable interphase. The requisite for an interphase to be described as ideally polarizable is that no charge flows through it [11].

Since anion and hydrogen adsorptions imply charge transfer, according to the processes:

$\mathrm{H}^{+}+\mathrm{Pt}+\mathrm{e} \rightleftharpoons \mathrm{Pt}-\mathrm{H}$

$A^{z-}+P t \rightleftharpoons P t-A^{x-}+(z-x) e$

the interphase in contact with platinum group metals cannot be considered as ideally polarizable in a strict sense. However, because the charge that flows remains localised on the surface of the electrode in the form of adsorbed species, thermodynamic equations can be easily extended to this case, just by the introduction of the notion of the total charge[12-14]. In this way, total charge includes both the charge involved on the adsorption processes and the true electronic charge that remains as an excess or defect of electrons on the metal, compensated by an equivalent ionic charge on the electrolyte side. The latter will be called in the following free charge.

A consequence of the previous definitions is that there are two different potentials of zero charge, the potential of zero total charge (pztc) and the potential of zero free charge (pzfc). Because usual voltammetric and potentiostatic measurements cannot separate adsorption from free charge, determination of the latter is more elusive and requires extra thermodynamic assumptions.

A suitable strategy to separate adsorption from capacitive processes is from their different time scale. Adsorption requires bond breaking and formation and therefore is expected to be slower than pure capacitive processes. For that reason, the response measured at short times to a fast perturbation of the interphase should allow determination of the free charge. This strategy has been used with the help of impedance spectroscopy measurements at very high potential modulation frequencies $[15,16]$. However, one limitation common to any potential controlled technique is the existence of a solution resistance that limits the time scale of the potential modulation.

An alternative approach is to perform a coulostatic perturbation of the interphase[17]. In this case, because there is no current flowing, the limitation of the uncompensated solution resistance to the time constant is avoided. This is the idea of the temperature jump method. In this technique, the interphase, initially at equilibrium, is subject to a sudden change of the temperature. The change of the open circuit potential due to the temperature change contains thermodynamic information on the interphase, which, under some circumstances, can be decoupled from adsorption processes. To achieve a very fast temperature change a high power laser can be used. By using a Q-Switched Nd:YAG laser producing $5 \mathrm{~ns}$ laser pulses of green light (532 nm) and 1-3 mJ/pulse, a temperature change of 20 degrees can be achieved. It has been demonstrated that adsorption processes can be separated from the double layer response in this way.

In this paper we revise different methods for the determination of total charge curves of platinum single crystal electrodes and we describe the effect of $\mathrm{pH}$ on such curves and, more particularly, on the potential of zero charge. Moreover, we discuss methodologies for the 
estimation of the position of the potential of zero free charge. For Pt(111) in the absence of anion specific adsorption, hydrogen and hydroxyl adsorption processes take place in well separated potential regions. In this case, there is a potential region where total and free charge coincide, allowing estimation, by extrapolation, of the potential of zero free charge. Values of the potential of zero free charge obtained in this way are compared with the results of the temperature jump measurement.

\section{The $\mathrm{CO}$ displacement experiment for total charge determination:}

The measure of voltammetric currents allows easy determination of the differential capacity (the low frequency or equilibrium differential capacity) provided the system behaves as reversible at the scan rate of the experiment. This capacity includes also the contribution from adsorption processes and, for that reason, it is often also called pseudocapacity. However, integration to determine the total charge from voltammetric currents requires knowledge of an integration constant. It has been shown that the charge displacement with $\mathrm{CO}[14$, 18]allows easy determination of total charge values that can be used for the integration of voltammetric currents.

In this experiment, $\mathrm{CO}$ is introduced into the atmosphere of the cell while the potential of the electrode is maintained constant[14, 18-20]. Adsorption of CO significantly decreases the electrical capacity of the interphase resulting in a flow of charge. The total charge that flows during the whole experiment can be considered equal to the difference between the charge at the end, $q^{C O}(E)$, and that at the beginning, $q(E)$, at the potential of the experiment.

$q_{d i s}(E)=q^{C O}(E)-q(E)$

Moreover, the total charge at the end of the experiment, $q^{C O}(E)$, is very low and can be considered negligible for most purposes. According to this, the $\mathrm{CO}$ displacement experiment is able to provide the total interfacial charge at the potential of the experiment. A complete curve giving the total charge as function of potential can be obtained from the integration of the voltammogram according to:

$q(E)=\int_{E^{*}}^{E} \frac{j}{v} d E+q^{C O}\left(E^{*}\right)-q_{d i s}\left(E^{*}\right)$

Where $E^{*}$ is potential of the $\mathrm{CO}$ displacement experiment. In this way, from the voltammetric current and a single charge displacement experiment, the full curve of charge versus potential can be obtained. Total charge values can be read from the curve even at potentials where the CO displacement cannot be performed because it is oxidised. The pztc can be read as the intercept with the $q=0$ axis.

\section{Model for the calculation of the pzfc of Pt(111)}

As previously discussed, the free charge is in general not directly available from voltammetric measurements. However, for the particular case of $\mathrm{Pt}(111)$ in solutions of anions that do not adsorb specifically (such as $\mathrm{ClO}_{4}{ }^{-}$or $\mathrm{F}^{-}$), it is usually assumed that hydrogen and hydroxyl adsorption regions are separated by a potential region where only capacitive processes take 
place. In this region, if there are no adsorption processes, total and free charge coincide, and, under this assumption, free charge values in this region can be obtained from the total charge curves mentioned above. It has been shown previously how this region can be extrapolated into the hydrogen region to obtain the pzfc[14, 21-23]. This strategy, which is only applicable to $\mathrm{Pt}(111)$ and not to the other basal planes, is illustrated in Figure 1. Also, in this figure, we can see that consideration of the term $q^{\mathrm{CO}}$ discussed above, while having little influence on the determination of the pztc, it makes a strong difference on the pzfc. This was initially pointed out in ${ }^{[24]}$

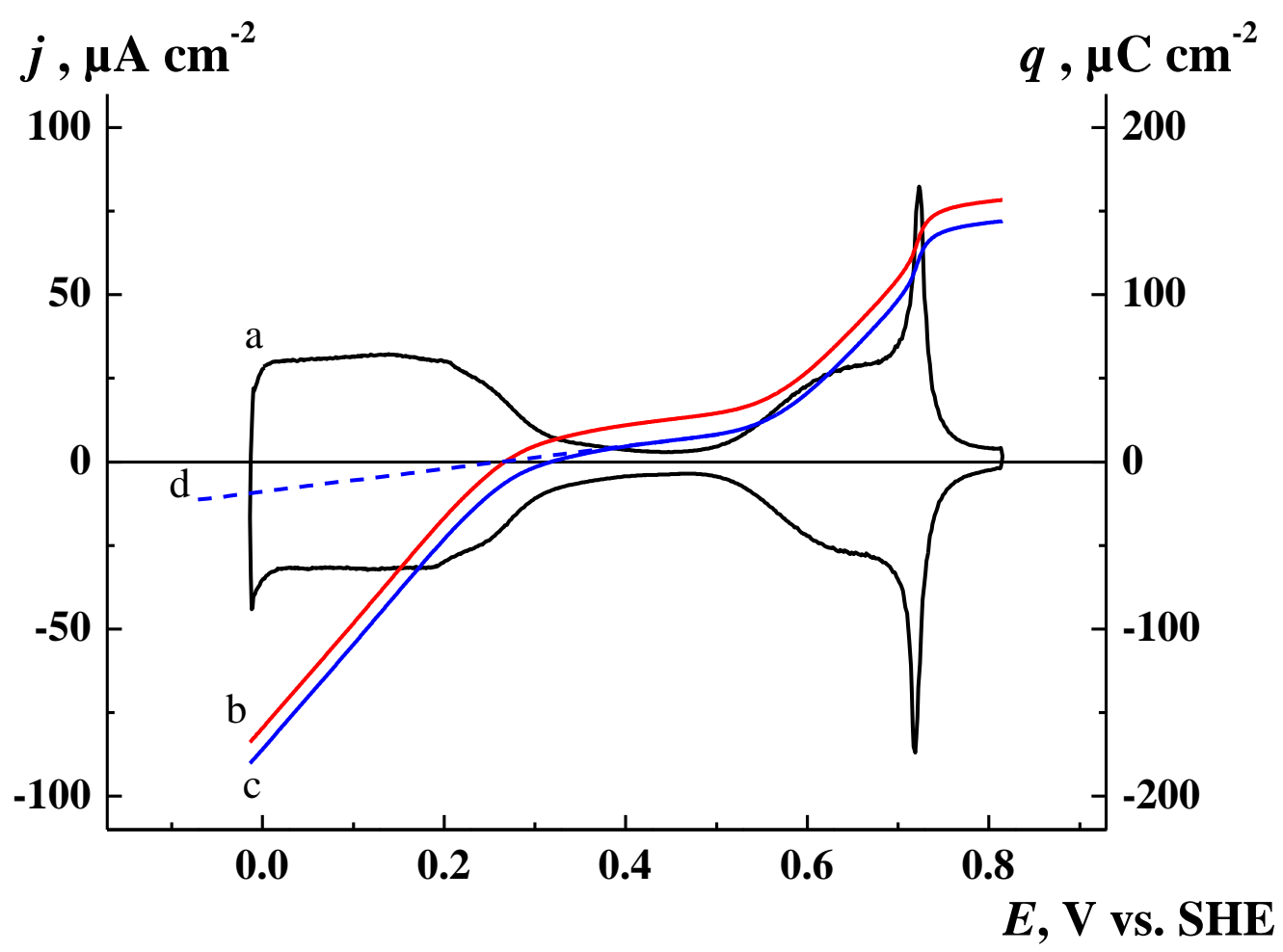

Figure 1: cyclic voltammogram (a, left axis) and total charge curve (b,c right axis) for a Pt(111) electrode in a $0.1 \mathrm{M}$ $\mathrm{HClO}_{4}$ solution. The extrapolation of the charge from the double layer region (curve d) illustrates the procedure to obtain the potential of zero free charge. Adapted with permission from [22]

\section{Effect of the pH}

Figure 2 shows the cyclic voltammograms and total charge curves for a $\mathrm{Pt}(111)$ electrode in solutions of different $\mathrm{pH}$. Figure $2 \mathrm{~A}$ shows the changes in the voltammogram when the $\mathrm{pH}$ is changed in the acidic end while Figure $2 \mathrm{~B}$ show voltammetric profiles in alkaline solutions. Acidic $\mathrm{pHs}$ are attained by mixing $\mathrm{HClO}_{4}$ and $\mathrm{KClO}_{4}$. On the other hand, alkaline $\mathrm{pHs}$ are obtained with mixtures of $\mathrm{NaOH}$ and $\mathrm{KClO}_{4}$. In both cases, the latter salt is used to maintain the ionic force constant at $0.1 \mathrm{M}$. As expected, hydrogen and hydroxyl adsorption regions shift negatively with the increase of $\mathrm{pH}$. Careful analysis of the curves reveals a small decrease of the hydrogen charge as $\mathrm{pH}$ increases as well as significant changes in the double layer region. On the acidic side, a broadening of the voltammetric profile in this region results in the formation of a new broad peak appearing at $0.36 \mathrm{~V}(\mathrm{SHE})$ as the $\mathrm{pH}$ is increased. The potential 
of this peak remains unaltered in the SHE scale and therefore overlaps with the hydroxyl region at $\mathrm{pH}>3$ as this region is shifted negatively in comparison with the more acidic $\mathrm{pHs}$. Following this argument, it can be concluded that at $\mathrm{pH}=1$ this peak in the double layer region is not visible because it is overlapped with the hydrogen region. The displacement of this process out of the hydrogen region as the $\mathrm{pH}$ is increased would explain the small decrease in hydrogen charge. On the other hand, the hydroxyl region also changes as a consequence of the $\mathrm{pH}$ increase. On acidic pHs this regions is composed of two peaks: a broad peak at lower potentials followed by a rather sharp peak. The charge on these peaks is redistributed as the $\mathrm{pH}$ is increased, with the broad peak increasing and the sharp peak decreasing. The existence of two peaks in this region has been tentatively assigned to $\mathrm{OH}$ adsorption from two sources [25]. Under this assumption, the broad peak would come from $\mathrm{OH}$ adsorption from structured water (through hydrogen bonding) while the sharp peak would be the result of unstructured water resulting from the perturbation of ions in the solution. On the side of alkaline solutions, double layer region is remarkably narrow, with a differential capacity of ca. $30 \mu \mathrm{Fcm}{ }^{-2}$ while hydroxyl region is composed of a single peak. In this $\mathrm{pH}$ region, hydroxyl adsorption would come from hydroxyl anions in solution, not from water as in acidic $\mathrm{pH}$, explaining the different morphology of this region depending on the $\mathrm{pH}$.

Accordingly to the shifts described above, total charge curves also shift negatively with the increase of the $\mathrm{pH}$. These are shown in Figure $2 \mathrm{C}$ and $\mathrm{D}$. Total charges displaced by $\mathrm{CO}$ at $0.1 \mathrm{~V}$ $(\mathrm{RHE})$, i.e., close to the onset of hydrogen evolution, decrease slightly as the $\mathrm{pH}$ is increased, in accordance with the hydrogen charge integrated from the voltammogram. To obtain total charge from the $\mathrm{CO}$ displaced charges it is necessary to account for the charge remaining on the CO covered surface, according to equation (2). To calculate the latter we need knowledge of the pzc of this interface. This fact was first discussed by M.J. Weaver [24] who gave an estimation of this parameter based on work function measurements on clean $\mathrm{Pt}(111)$ surfaces and model interfaces created in UHV (by sequentially dosing the different components). The remaining charge on the $\mathrm{CO}$ covered surface on $\mathrm{Pt}(111)$ was latter measured for $0.1 \mathrm{M} \mathrm{HClO}_{4}$ by the immersion method [26], proving to be consistent with the pzc estimated from UHV measurement. Although the variation of this remaining charge with $\mathrm{pH}$ has not been measured experimentally, it is expected to increase if pzc of the $\mathrm{CO}$ covered surface does not change with $\mathrm{pH}$ and the range of potentials of interest is shifted negatively. The consequence of this correction is that, while displaced charge (at the same RHE potential) is nearly constant, total charge curves are displaced negatively resulting in an intercept at $q=0$ (pztc) that has moved into the hydroxyl region (compare Figure $2 \mathrm{C}$ and $\mathrm{D}$ ).

Values of pztc resulting from such intercepts are plotted as a function of $\mathrm{pH}$ in Figure 3. Two clearly distinct trends are observed. For acidic $\mathrm{pHs}$, where pztc lies in the double layer region, pztc values shift very little with the $\mathrm{pH}$ (in the SHE scale, the shift nearly $59 \mathrm{mV} /$ decade in the RHE scale). On the other side, for an alkaline solution, the pztc lies in the hydroxyl region and shift nearly $59 \mathrm{mV} /$ decade in the SHE scale (close to zero in the RHE scale). 

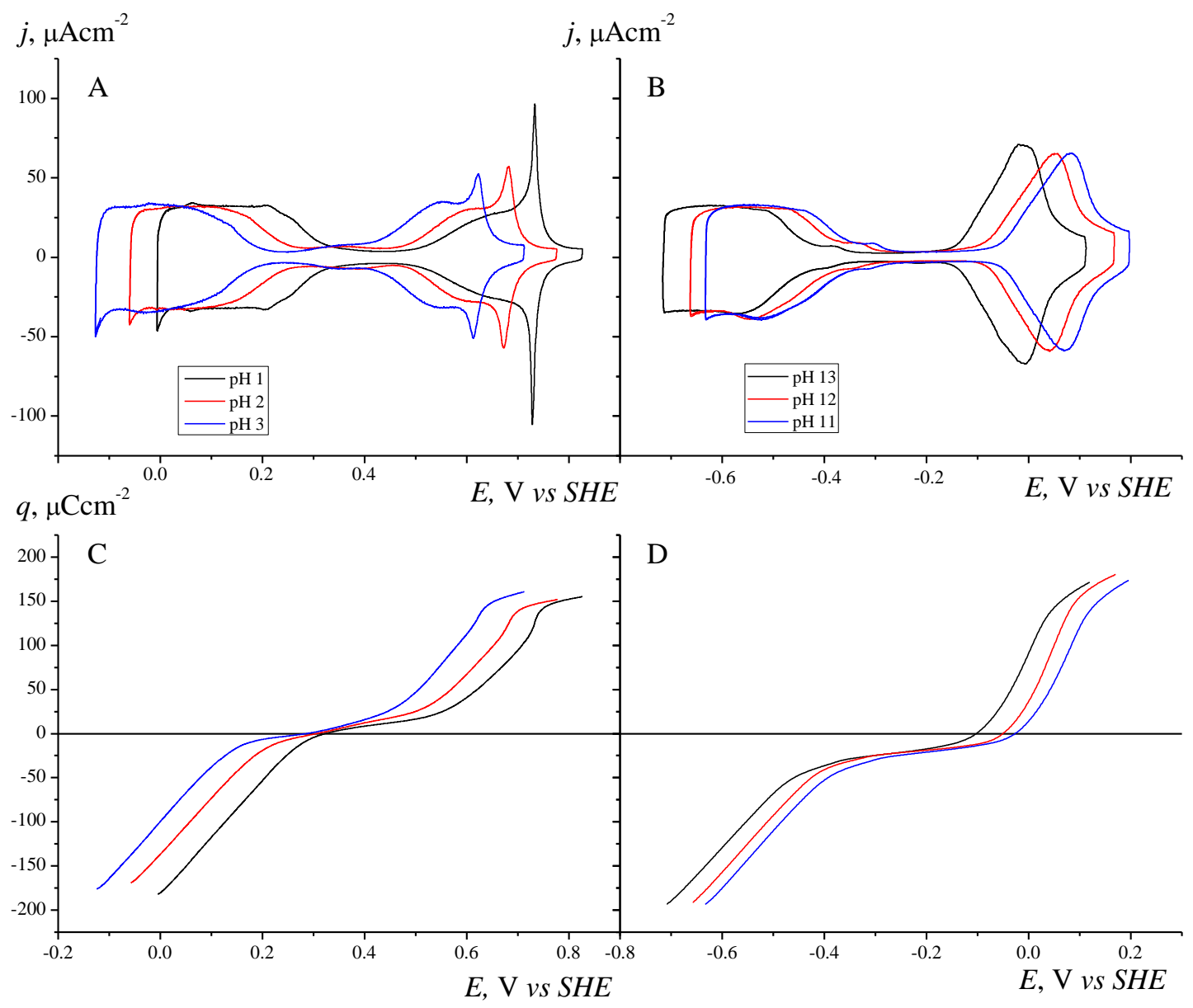

Figure 2: Cyclic voltammograms (A, B) and total charge curves (C, D) for $\mathrm{Pt}(111)$ in solutions of different $\mathrm{pH}$ (mixtures of $\mathrm{KClO}_{4}{ }^{\prime} \mathrm{HClO}_{4}$. and $\mathrm{NaOH}$ with total perchlorate concentration of $0.1 \mathrm{M}$ ). A, C) pH=1, 2, and 3; $\mathrm{B}, \mathrm{D}$ ) $\mathrm{pH}=11,12$ and 13. Scan rate: $50 \mathrm{mV} / \mathrm{s}$ 


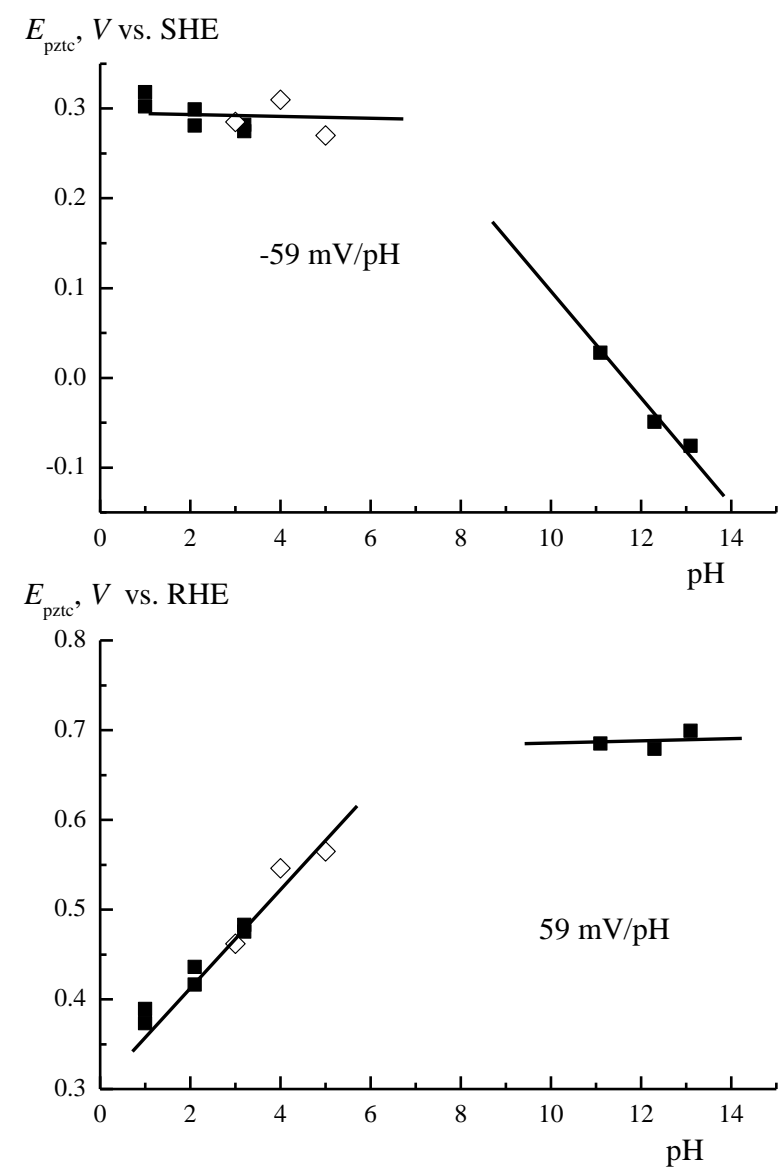

Figure 3: Values of pztc as a function of $\mathrm{pH}$, ad determined from the combination of $\mathrm{CO}$ displacement and voltammetric charges. All values are corrected to consider the remaining charge on the CO covered surface. Closed symbols corresponds mixtures of $\mathrm{KClO}_{4^{\prime}} \mathrm{HClO}_{4}$. and $\mathrm{NaOH}$ with total perchlorate concentration of $0.1 \mathrm{M}$. Open symbols corresponds to mixtures of $\mathrm{NaF}$ and $\mathrm{HClO}_{4}$.

Two issues need to be addressed. First it would be desirable to have more data available in the $\mathrm{pH}$ region close to neutrality. Voltammogram a) in Figure 4 illustrate how this $\mathrm{pH}$ region is not attainable in unbuffered solutions. Since hydrogen and hydroxyl adsorption involve consumption or release of $\mathrm{H}^{+}$to the solutions, they cause a strong change in the interfacial $\mathrm{pH}$ resulting in a distorted voltammogram unless a buffer is present to attenuate this effect. The problem with most common buffer solution is that they are composed of anions that adsorb specifically on the surface of platinum electrodes, therefore hampering the comparison with results in perchlorate or $\mathrm{NaOH}$ solutions. The only anion from a weak acid that has been proved that does not adsorb specifically is fluoride. The proof comes from the similarity of the $\mathrm{CV}$ in solutions of $\mathrm{HF} / \mathrm{NaF}$ and mixtures of $\mathrm{KClO}_{4} / \mathrm{HClO}_{4}$ [27-29]. However, working with $\mathrm{HF}$ is particularly dangerous and, what is more important, $\mathrm{HF}$ commercially available is usually of not enough purity for such interfacial analysis. We have recently demonstrated that buffer solutions suitable for working at $\mathrm{pH}$ values close to neutrality can be prepared by mixing $\mathrm{NaF}$ and $\mathrm{HClO}_{4}$ resulting in an electrolyte solution of sufficiently high purity and absent of specific adsorption phenomena on platinum [23]. While high HF concentration can lead to dissolution of the glass cell in some extent, which may lead to the contamination of the solution, we found 
that, for the concentrations used in this study, this effect poses no problem. In all cases cleanliness of the solution was tested from the stability of the cyclic voltammogram. Figure 4B demonstrates the effect of the presence of fluoride on the voltammetric profile at $\mathrm{pH} 5$. HF is a weak acid with pKa close to 3 and therefore the HF/F- equilibrium act as a buffer. It is clear that the buffering effect associated to the presence of $\mathrm{HF}$ allows to recover the usual voltammetric shape for Pt(111) even in solutions with $\mathrm{pH}$ close to neutral.

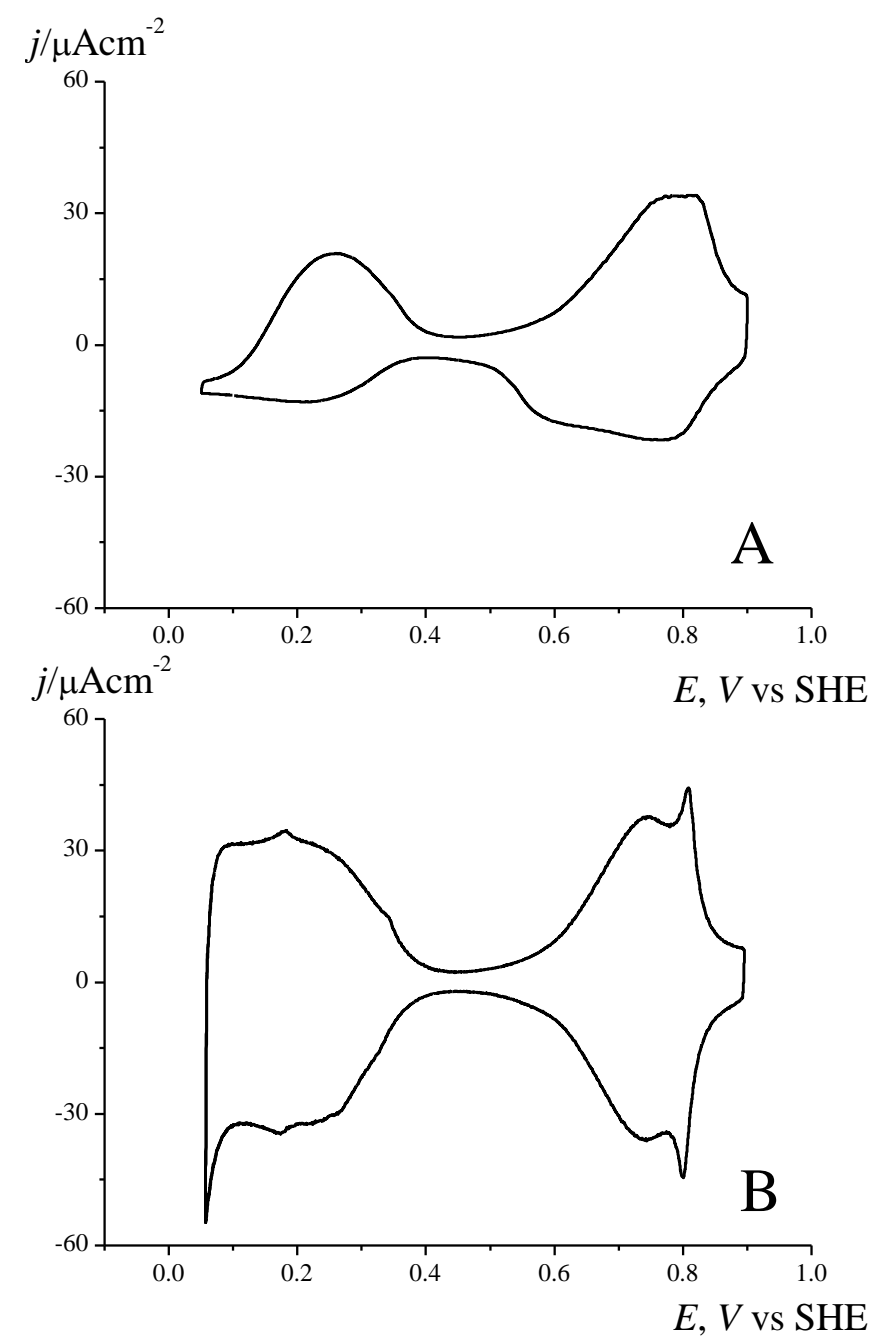

Figure 4: Comparison of Voltammograms for $\mathrm{Pt}(111)$ at pH 5 in $\mathrm{A}) \mathrm{HClO} 4+\mathrm{KClO} 4$ mixtures; $\mathrm{B}) \mathrm{HClO}_{4}+$ $\mathrm{NaF}$ mixtures. Scan rate $50 \mathrm{mV} / \mathrm{s}$.

The voltammograms of Figure 5 show the effect of $\mathrm{pH}$ variation on the voltammetric profile. The voltammogram at $\mathrm{pH}=3$ is almost identical to the one obtained in the absence of fluoride, demonstrating that this anion does not adsorb specifically on this surface. The similarity with the $\mathrm{CV}$ recorded in the absence of HF demonstrates that presence of this acid does not lead, under the present conditions, to significant contamination of the solution from glass dissolution. The voltammograms on this figure demonstrate the trends already described for Figure $2 \mathrm{~A}$. The broad wave in the double layer region can be observed at $\mathrm{pH}=3$ but it significantly overlaps with the currents due to hydroxyl adsorption at $\mathrm{pH}=4$. At $\mathrm{pH}=5$ this process is no longer visible and only a very narrow double layer region separates the hydrogen adsorption from hydroxyl adsorption region. The other trend already mentioned is the 
redistribution of charges within the hydroxyl adsorption region, resulting in an increase of the broad peak that precedes the sharp spike and a significant decrease of the height of the latter.

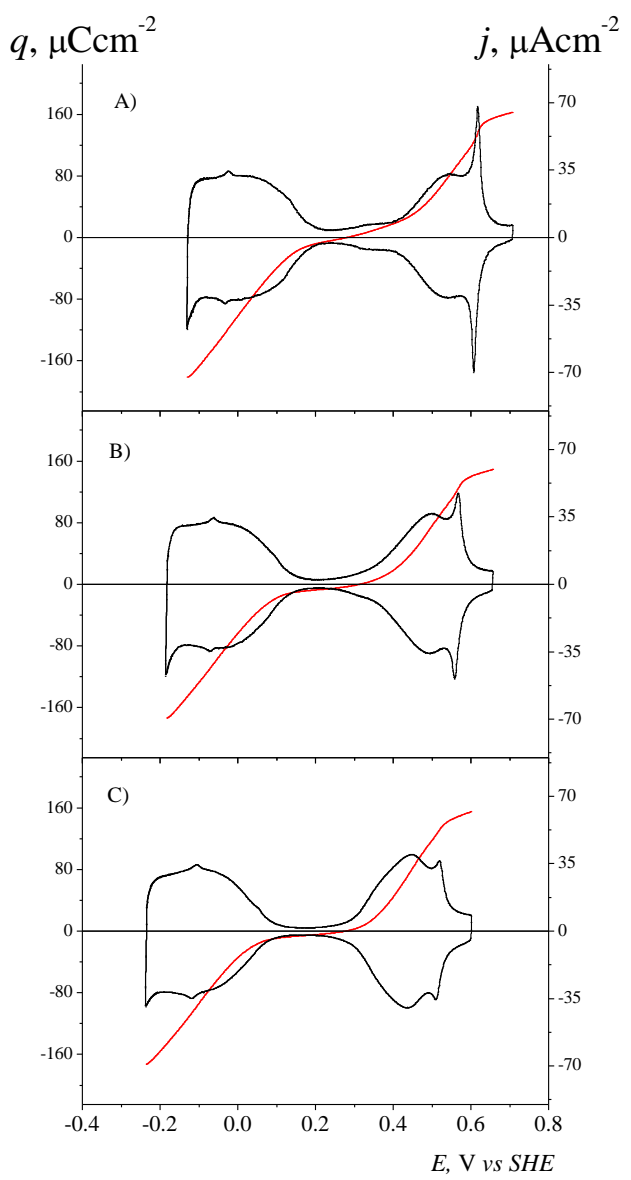

Figure 5: Cyclic Voltammograms (black, right-hand axis) and total charge curves (red, left-hand axis) for $\mathrm{Pt}(111)$ in $\mathrm{A}$ ) $\mathrm{pH}=3, \mathrm{~B}) \mathrm{pH}=4$ and $\mathrm{C}$ ) $\mathrm{pH}=5$. Solutions prepared by mixing $\mathrm{HClO}_{4}$ and $\mathrm{NaF}$. Scan rate $50 \mathrm{mV} / \mathrm{s}$.

Careful inspection of the corresponding total charge curves represented in Figure 5 reveals the following interesting trend. For $\mathrm{pH}$ lower than 3 the pztc lies in the onset of the hydrogen adsorption region. This indicates that pzfc should lie at potentials more negative than the pztc. On the other hand, for more alkaline pHs, the pztc lies at the onset of hydroxyl adsorption and double layer charge is negative. The consequence of this is that pzfc should be located at potentials higher than the pztc. At $\mathrm{pH}=3 \mathrm{pztc}$ and pzfc coincide in the double region. We have previously proposed that this $\mathrm{pH}$ has a particular meaning that can be identified with the pK of adsorbed water[22].

The second issue that deserves further attention is that of the correction for the remaining charge after $\mathrm{CO}$ adsorption. This correction might be questioned because is based on a measurement done at a single $\mathrm{pH}[26]$ or even worst on an estimation based on ex situ measurements[24]. Some of the trends discussed above results from the increase of the term $q^{\mathrm{CO}}$ as the absolute potential scale shift negatively with the increase of the $\mathrm{pH}$. In this regards, while displaced charge at $0.1 \mathrm{~V}(\mathrm{RHE})$ is nearly constant or even decrease when the $\mathrm{pH}$ is increased, the total charge curves in Figure 2 and Figure 5 are shifted to more negative values as a consequence of the term $q^{\mathrm{cO}}$. Therefore, it is convenient to devise a method to test the validity of such trends. We have previously demonstrated that strong anion adsorption, such 
as chloride, can be used to block hydroxyl adsorption at high potentials [30, 31]. Under such conditions, the properties of the interphase should become $\mathrm{pH}$ independent in the potential region where the surface is covered by the anion. Indeed, comparison of cyclic voltammograms recorded under the presence of chloride at different $\mathrm{pHs}$ reveals the coincidence of the profiles in the region of chloride adsorption as exemplified in Figure 6 . The peak at $0.52 \mathrm{~V}$ in the curve corresponding to the higher $\mathrm{pH}$ is probably due to the replacement of chloride by hydroxyl as the potential is scanned positively. This peaks shifts to higher potentials if $\mathrm{pH}$ is decreased. In the potential region between hydrogen adsorption and this peak, the surface remains covered by chloride and for that reason, voltammetric current is independent of $\mathrm{pH}$, being possible to assume that total charge should also be invariant with $\mathrm{pH}$ in this region. This provides a method for establishing the relative position of the total charge curves of different pHs, by making them coincide in this potential region. In this way, by knowing the integration constant (by a $\mathrm{CO}$ displacement at a single potential) in just one pH value, it is possible to calculate the absolute position of all the other total charge curves at a different $\mathrm{pHs}$. From these curves, it is possible to read the total charge values at a given potential and to compare these with the displaced charge by $\mathrm{CO}$ at the same potential. The difference between the two charge values will be the term $q^{\mathrm{CO}}$ that can be calculated for the different $\mathrm{pH}$ values in this way. The resulting values of $q^{\mathrm{CO}}$ are shown as a function of $\mathrm{pH}$ in Figure 7. This figure shows the $q^{\mathrm{CO}}$ values measured at a constant potential in the RHE scale $(0.1 \mathrm{~V} \mathrm{RHE})$. In this case, the increase in the remaining charge is due to the displacement of the absolute potential to more negative values as the $\mathrm{pH}$ increases. Calculated values from the $\mathrm{pzc}$ value measured at $\mathrm{pH}=1[24,26]$, considered as constant with $\mathrm{pH}$, are also included in Figure 7 . The coincidence between calculated and measured values support the assumption that pzc of the $\mathrm{CO}$ covered surface is $\mathrm{pH}$ independent. It should be pointed out that, because the interphase should not be affected by the presence of the chloride in the low potential region, particularly at $0.1 \mathrm{~V} \mathrm{RHE}$, displaced charges at this potential should be independent of the chloride presence. For that reason, displaced charges in the absence of chloride have been used to calculate $q^{\mathrm{CO}}$ values in Figure 7 . 


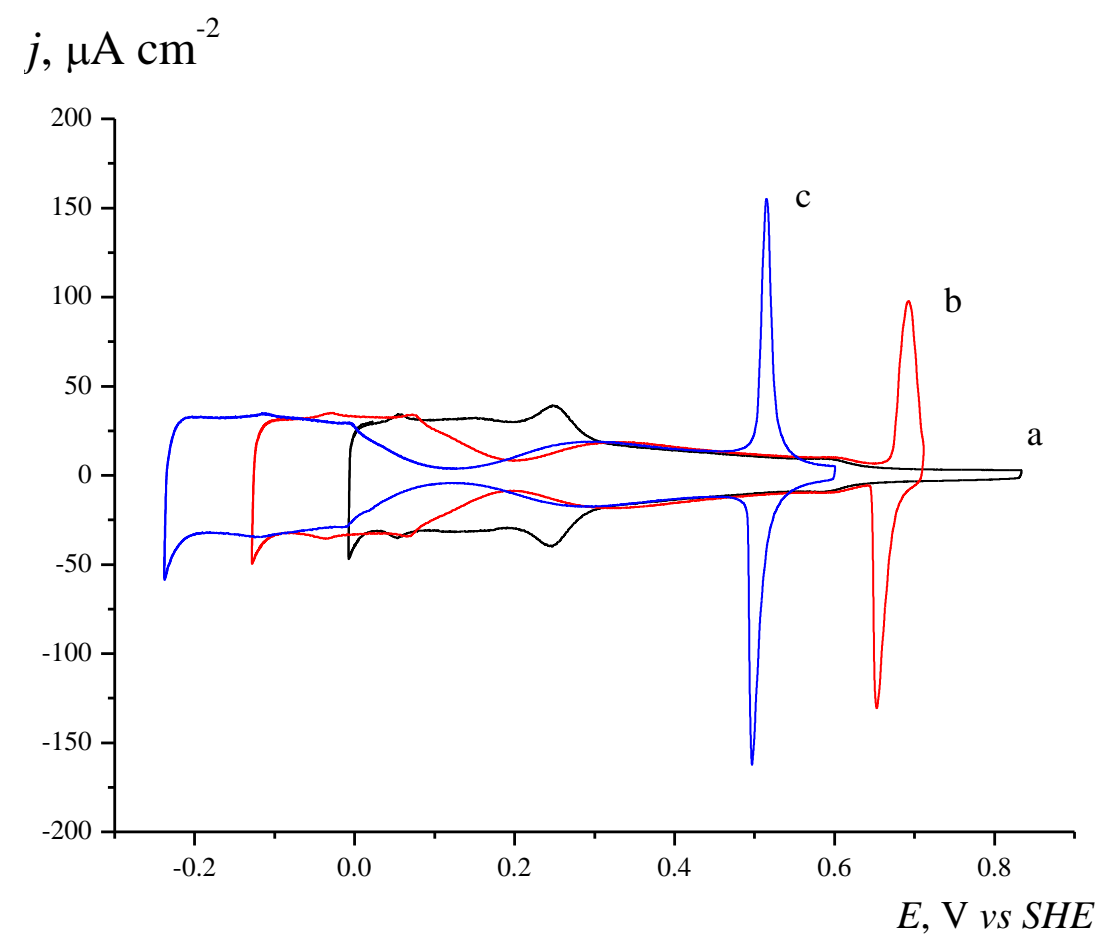

Figure 6: Cyclic Voltammograms recorded at pH 1 (a, black), 3 (b, red) and 5 (c, blue) in $\mathrm{HClO}_{4} / \mathrm{NaF}$ mixtures containing $1 \mathrm{mM}$ chloride. Scan rate: $50 \mathrm{mV} / \mathrm{s}$.

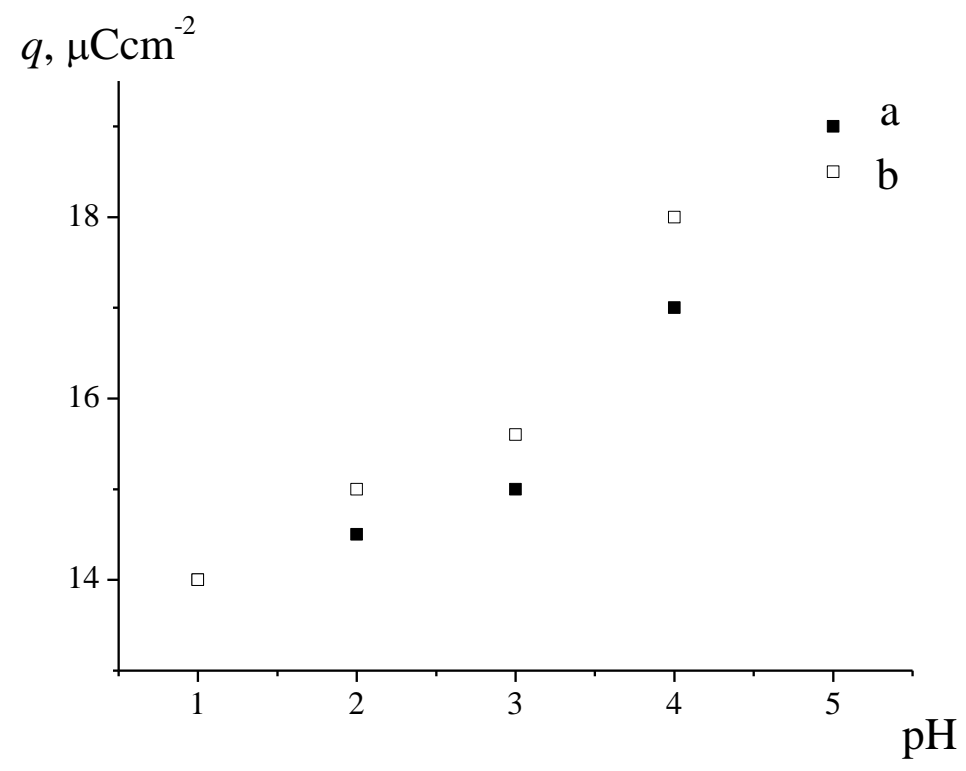

Figure 7. Plot as a function of the $\mathrm{pH}$ of the remaining charge on the $\mathrm{CO}$ covered $\mathrm{Pt}(111)$ surface in solutions of different $\mathrm{pH}$ a) (closed symbols) measued at $0.1 \mathrm{~V} \mathrm{~b}$ ) calculated from the pzc value given in [24]

\section{Results of the temperature jump measurements}

The change in the open circuit potential during a change of temperature induced by pulsed laser irradiation can be rationalised considering that the main contribution comes from the perturbation of the dipolar part of the potential drop [32-35]. Such dipolar contribution will be negative for positive electrode charges, since, in this case, the water dipoles will be oriented with the oxygen towards the metal adding a negative term to the potential difference $\phi^{\mathrm{M}}$ - $\phi^{\mathrm{s}}$. 
Disordering this dipole layer will decrease this negative contribution resulting in a positive shift of the potential. Conversely, negative charges orient the water with the hydrogen towards the metal giving a positive contribution to the potential drop. In this case, heating decreases the potential, resulting in a negative transient. Other contributions to the potential change come from the variation of the spillover of electrons during temperature change and from the thermodiffusion potential in the solutions. It has been shown that the latter contributions can be considered negligible [32], therefore the sign of the laser induced potential transients are indicative of the orientation of the dipoles at the interfase. Then, the potential of zero transient coincides with the turnover of the direction of the dipoles induced by the potential. Orientation of water dipoles depends strongly on the electric field and therefore is a property linked with the true electronic charge at the interphase. While this potential of zero transient does not necessarily need to coincide with the potential of zero free charge, because chemical interaction may also play a role in the orientation of water dipoles, the cases where both magnitudes are available to show that they are very close. This is the case of $\mathrm{Au}(111)$ [33] and also, as we will show later, of $\mathrm{Pt}(111)$. In conclusion, identification of the potential of zero transient can be used to localize the potential of zero free charge.

In addition to the previous description, thermodynamic considerations demonstrate that the thermal coefficient of the potential drop is linked with the entropy of double layer formation through $[32,33,35,36]$ :

$\left(\frac{\partial \Delta_{M}^{S} \Phi}{\partial T}\right)_{q}=-\left(\frac{\partial \Delta S}{\partial q}\right)_{T}$

Where $q$ is the total charge, and $\Delta S$ is the entropy of formation of the interphase, defined as the difference between the excess entropy of the interphase and the entropy of the components of the interphase when they are forming part of the bulk phases [37]. $\Delta_{M}^{S} \Phi$ is the potential drop at the interphase, between the metal and the solution phases. According to this equation, the potential of zero transient coincides with the potential of maximum entropy (pme), i.e., maximum disorientation of water dipoles, if we consider that water is the main component of the interphase. 

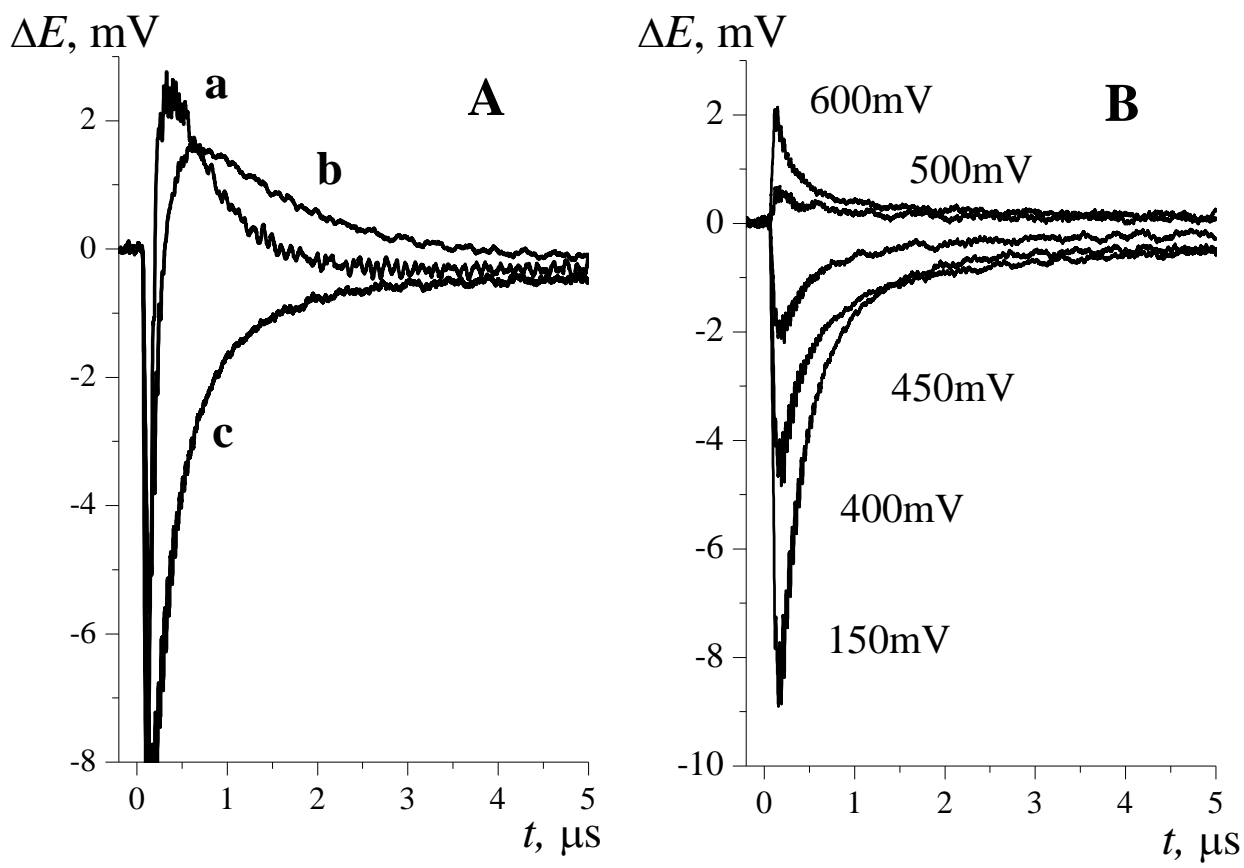

Figure 8: Laser potential transients at A) $150 \mathrm{mV}$ vs RHE at different pH ca. a) 1, b) 2 and c) 3; B) pH=3 at different applied potentials, as labelled. (0.1-x) $\mathrm{M} \mathrm{KClO}_{4}+x \mathrm{M} \mathrm{HClO}_{4}$

Some potential transients obtained for $\mathrm{Pt}(111)$ are shown in Figure 8. Figure $8 \mathrm{~A}$ shows potential transients obtained at $0.15 \mathrm{~V}$ RHE in solutions of different $\mathrm{pH}$ values. Figure $8 \mathrm{~B}$, shows the transients at $\mathrm{pH} 3$ measured at different potential values. While the transients obtained at higher pHs follow monotonously the shape of the temperature change, those obtained at acidic $\mathrm{pHs}$ in the hydrogen region exhibit a bipolar shape. Such behaviour has been explained considering the kinetics of the reaction of hydrogen adsorption [32, 35]. The change of the potential, induced by the temperature increase, forces a variation on the hydrogen coverage. Because such change of coverage takes place coulostaticaly, it implies also a change in the free charge that will cause, in turn, also a change of potential. However, the adaptation of the hydrogen coverage to the new potential is slow (for the time scale of the experiment), resulting on a response with longer time constant. Moreover, the rate of this reaction decreases with the increase of the $\mathrm{pH}$ and therefore, hydrogen adsorption and reorientation of water dipoles are almost completely decoupled at $\mathrm{pH}=3$. It has been shown previously that a value of the rate constant for hydrogen adsorption can be estimated from the shape of the curve measured at $\mathrm{pH}=1[32]$.

Transients measured at $\mathrm{pH}=3$ show a monotonous response. A simple model for the heat transfer at the interfase shows $[32,33]$ that, except for very short times, the temperature decays as a function of the $t^{-1 / 2}$. Plot of the potential transients as a function of $t^{-1 / 2}$ show that potential varies proportionally to the temperature change, i.e., the time constant of the response is shorter than the time scale of the measurement. From the proportionality constant the thermal coefficient of the potential drop, $\left(\partial \Delta_{M}^{S} \Phi / \partial T\right)_{q, \mu_{i}, \Gamma_{i}}$, could be obtained if the magnitude of the temperature change is known (The subscript $\Gamma_{1}$ is added to the previous partial derivative to stress that coverage of adsorbed species is constant during the measurement under the present conditions because the adsorption processes are too slow to 
respond in this time scale). However, the magnitude of the temperature change is not known with sufficient accuracy and therefore, the most important piece of information extracted from these potential transients is their sign, which, according to the previous discussion corresponds to the sign of the dipolar contribution. Figure $8 \mathrm{~B}$ shows that potential transients recorded in the lower limit of potentials are negative, and that they progressively become positive as the potential is increased. The laser transient reverse sign at around $0.47 \mathrm{~V}$ indicating that this is the location of the pme. This potential value is very close to the pztc determined above. Because, as pztc and pzfc coincide at this $\mathrm{pH}$, we conclude that pme is located very close to the pzfc, as discussed above.

A similar situation is obtained for the other basal planes. A kinetic response (non-monotonous bipolar shape) is obtained at the more acidic pHs and a linear response at higher pHs. Even if there are kinetic complications to the interpretation of the potential transient, the potential of the inversion of the transient can in general be determined even at the more acidic $\mathrm{pH}$. Values of the pme are compared with the pztc values for the three basal planes, as a function of $\mathrm{pH}$, in Figure 9. While pztc and pme are very close for Pt(111), they differ significantly for Pt(100) and $\mathrm{Pt}(110)$. In general, pme values are located at lower potentials than the pztc. As discussed above, at the pztc, if it lies within the hydrogen adsorption, positive free charge is compensated with the hydrogen adsorption charge. Therefore pzfc $<p z t c$. This explain the relative position between pme and pztc and reinforces the idea that pme gives a good estimation of the location of the pzfc, in fact, the best estimation we have so far for Pt(100) and Pt(110).

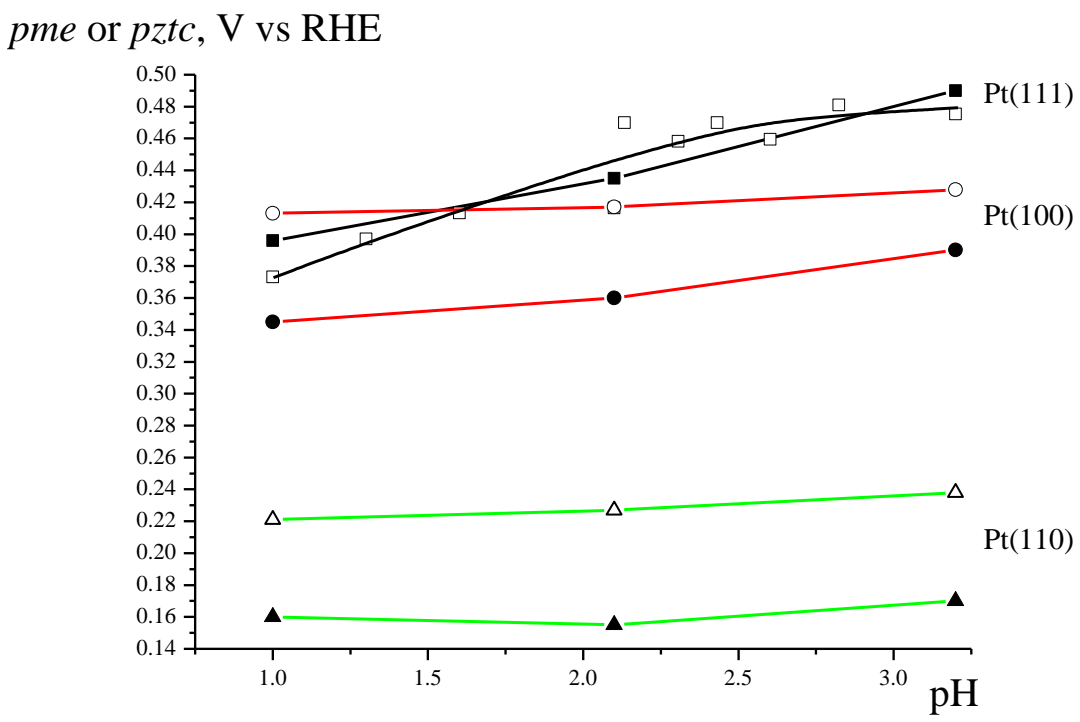

Figure 9: Dependence of pme (filled symbols) and pztc (open symbols) with pH for the three basal planes.( $\square, \square)$ $\operatorname{Pt}(111),(0,0) \operatorname{Pt}(100)$ and $(\Delta, \Delta) \operatorname{Pt}(110)$

\section{Conclusions:}

The application of the $\mathrm{CO}$ displacement method to the investigation of the interfacial properties of platinum single crystal electrodes has been revised and the effect of $\mathrm{pH}$ on the potential of zero total charged analysed. For Pt(111), advantage can be taken of the fact that hydrogen and hydroxyl adsorption region are well separated. Under the assumption that only free charge resides on the interphase between those regions, a simple extrapolation 
procedure can be used to extract values of the potential of zero free charge, to study the effect of the $\mathrm{pH}$ on this parameter. Emphasis is done on the neutral $\mathrm{pH}$ region, where a buffer solution of novel composition is used to avoid specific adsorption of anions while preserving a sufficient degree of cleanliness. This buffer is achieved with mixtures of $\mathrm{NaF} / \mathrm{HClO} 4$.

The study in a broad range of $\mathrm{pH}$ shows that values of pztc are affected by the interpherence of hydroxyl adsorption region for $\mathrm{pH}>7$. As a consequence, while pztc is nearly invariable with $\mathrm{pH}$ (in the SHE scale)up to $\mathrm{pH} 6$, in the alkaline end, pztc values shift nearly $60 \mathrm{mV} / \mathrm{pH}$ unit (in the SHE scale). On the other hand, the extrapolated values of the pzfc remain nearly invariant even for the alkaline solutions.

For the other basal planes, free charge values are not accessible. On the other hand, the laser induced temperature jump method provides indication of the sign of the dipolar contribution to the potential. This is intimately related with the electric field at the interphase and therefore related with the free charge. The potential where the dipoles change sign, the pme, give an indication of the position of the pzfc. The good coincidence between pme and pzfc for $\mathrm{Pt}(111)$ and $\mathrm{Au}(111)$ [33] support this idea. Value of the pme, as a function of $\mathrm{pH}$ have been given for the three basal planes of platinum.

Acknowledgments:

Support from MINECO (Spain) through project CTQ2016-76221-P and Generalitat Valenciana through project PROMETEOII/2014/013 is greatly acknowledged. RMH thankfully acknowledges support from Generalitat Valenciana under the Santiago Grisolia Program (GRISOLIA/2013/008). P. Sebastian also knowledges MECD for the award of a FPU grant.

\section{References:}

1. Trasatti, S. and Lust, E., The potential of zero charge, in: Mod. Aspects Electrochem., White, R. E., Bockris, J. O. M. and Conway, B. E. (Eds.)vol. 33. Kluwer Academic/Plenum Publishers, New York, 1999, pp. 1-215.

2. Frumkin, A. N., Petrii, O. A. and Damaskin, B. B., Potential of Zero Charge, in: Comprehensive Treatise of Electrochemistry, Bockris, J. O. M., Conway, B. E. and Yeager, E. (Eds.)vol. 1. Plenum, New York, 1980, pp. 221-289.

3. Bockris, J. O. M., Argade, S. D. and Gileadi, E., Electrochim. Acta, 1969, vol. 14, p. 12591283.

4. Hamelin, A., Double layer properties at sp and sd metal single-crystal electrodes, in: Modern Aspects of Electrochemistry, Conway, B. E., White, R. E. and Bockris, J. O. M. (Eds.)vol. 16. Plenum, New York, 1985, pp. 1-101.

5. Valette, G. and Hamelin, A., J. Electroanal. Chem., 1973, vol. 45, p. 301-319.

6. Hamelin, A. and Stoicoviciu, L., J. Electroanal. Chem., 1987, vol. 234, p. 93-105.

7. Hamelin, A., Stoicoviciu, L., Doubova, L. and Trasatti, S., Surf. Sci., 1988, vol. 201, p. L498-L506.

8. Lecoeur, J., Andro, J. and Parsons, R., Surf. Sci., 1982, vol. 114, p. 320-330.

9. Lecoeur, J., Bellier, J. P. and Koehler, C., J. Electroanal. Chem., 1992, vol. 337, p. 197216.

10. Lecoeur, J., Bellier, J. P. and Koehler, C., J. Electroanal. Chem., 1994, vol. 375, p. 117122. 
11. Parsons, R., Thermodynamic methods for the study of interfacial regions in electrochemical systems, in: Comprehensive Treatise of Electrochemistry, Bockris, J. O. M., Conway, B. E. and Yeager, E. (Eds.)vol. 1. Plenum, New York, 1980, pp. 1-44.

12. Frumkin, A. N., Balashova, N. A. and Kazarinov, V. E., J. Electrochem. Soc., 1966, vol. 113, p. 1011-1025.

13. Frumkin, A. N. and Petrii, O. A., Electrochim. Acta, 1975, vol. 20, p. 347-359.

14. Climent, V., Garcia-Araez, N., Herrero, E. and Feliu, J., Russ. J. Electrochem., 2006, vol. 42, p. 1145-1160.

15. Sibert, E., Faure, R. and Durand, R., J. Electroanal. Chem., 2001, vol. 515, p. 71-81.

16. Kerner, Z. and Pajkossy, T., Electrochim. Acta, 2002, vol. 47, p. 2055-2063.

17. Smalley, J. F., Krishnan, C. V., Goldman, M., Feldberg, S. W. and Ruzic, I., J. Electroanal. Chem., 1988, vol. 248, p. 255-282.

18. Climent, V., Gómez, R. and Feliu, J. M., Electrochim. Acta, 1999, vol. 45, p. 629-637.

19. Clavilier, J., Albalat, R., Gómez, R., Orts, J. M., Feliu, J. M. and Aldaz, A., J. Electroanal. Chem., 1992, vol. 330, p. 489-497.

20. Clavilier, J., Albalat, R., Gómez, R., Orts, J. M. and Feliu, J. M., J. Electroanal. Chem., 1993, vol. 360, p. 325-335.

21. Climent, V., Gómez, R., Orts, J. M., Aldaz, A. and Feliu, J. M., The potential of zero total charge of single-crystal electrodes of platinum group metals, in: The Electrochemical Society Proceedings (Electrochemical Double Layer), Korzeniewski, C. and Conway, B. E. (Eds.)vol. 97-17. The Electrochemical Society, Inc., Pennington, NJ, 1997, pp. 222 237.

22. Rizo, R., Sitta, E., Herrero, E., Climent, V. and Feliu, J. M., Electrochim. Acta, 2015, vol. 162, p. 138-145.

23. Martinez-Hincapie, R., Sebastian-Pascual, P., Climent, V. and Feliu, J. M., Electrochem. Commun., 2015, vol. 58, p. 62-64.

24. Weaver, M. J., Langmuir, 1998, vol. 14, p. 3932-3936.

25. Berna, A., Climent, V. and Feliu, J. M., Electrochem. Commun., 2007, vol. 9, p. 27892794.

26. Cuesta, A., Surf. Sci., 2004, vol. 572, p. 11-22.

27. Wagner, F. T. and Ross, P. N., J. Electroanal. Chem., 1988, vol. 250, p. 301-320.

28. Nart, F. C., Iwasita, T. and Weber, M., Electrochim. Acta, 1994, vol. 39, p. 961.

29. Lachenwitzer, A., Li, N. and Lipkowski, J., J. Electroanal. Chem., 2002, vol. 532, p. 85-98.

30. Garcia-Araez, N., Climent, V., Herrero, E., Feliu, J. and Lipkowski, J., J. Electroanal. Chem., 2005, vol. 576, p. 33-41.

31. Garcia-Araez, N., Climent, V., Herrero, E., Feliu, J. M. and Lipkowski, J., Electrochim. Acta, 2006, vol. 51, p. 3787-3793.

32. Climent, V., Coles, B. A. and Compton, R. G., J. Phys. Chem. B, 2002, vol. 106, p. 59885996.

33. Climent, V., Coles, B. A. and Compton, R. G., J. Phys. Chem. B, 2002, vol. 106, p. 52585265.

34. Garcia-Araez, N., Climent, V. and Feliu, J. M., J. Am. Chem. Soc., 2008, vol. 130, p. 38243833.

35. Garcia-Araez, N., Climent, V. and Feliu, J., J. Phys. Chem. C, 2009, vol. 113, p. 92909304.

36. Benderskii, V. A. and Velichko, G. I., J. Electroanal. Chem., 1982, vol. 140, p. 1-22.

37. Harrison, J. A., Randles, J. E. B. and Schiffrin, D. J., J. Electroanal. Chem., 1973, vol. 48, p. 359-381. 
